Isidore Fleischer, Centre de Recherches Mathematiques, Universite de Montreal, Montreal, Quebec H3C 3P8, Canada

\title{
A VITALI-LIKE CONVERGENCE THEOREM FOR THE HENSTOCK INTEGRAL
}

\begin{abstract}
Theorem. If Henstock integrable $f_{n}$ converge in measure to a finite $f$ and their primitives $F_{n}$ are equi-ACG $G_{*}$ and converge pointwise to a continuous $F$ then $\int f=\lim F_{n}$.
\end{abstract}

Let's start by recalling some of the less current notions.

A real-valued function $f$ on $[a, b]$ is said to be Henstock (-Kurzweil) integrable if the sums, $\Sigma f\left(t_{i}\right)\left(v_{i}-u_{i}\right)$, for divisions of $[a, b]$ into non-overlapping intervals $\left[u_{i}, v_{i}\right]$ with tags $t_{i}$ satisfying $t_{i}-\delta\left(t_{i}\right) \leq u_{i} \leq t_{i} \leq v_{i} \leq t_{i}+\delta\left(t_{i}\right)$ for a given positive-valued function $\delta(t)$ on $[a, b]$, converge to a limit $\int_{a}^{b} f$ as $\delta(t)$ converges to zero along the down-directed pointwise ordered strictly positive functions. Function $f$ is then integrable on every subinterval, the indefinite integral or primitive $F$ being continuous and additive on non-overlapping subintervals, and one has for "partial divisions", i.e. consisting of non-overlapping subintervals with $t_{i}-\delta\left(t_{i}\right) \leq u_{i} \leq t_{i} \leq v_{i} \leq t_{i}+\delta\left(t_{i}\right), \Sigma\left|F\left(v_{i}\right)-F\left(u_{i}\right)-f\left(t_{i}\right)\left(v_{i}-u_{i}\right)\right| \rightarrow$ 0 as $\delta(t) \downarrow 0$ as above (Henstock's Lemma).

Function $F$ is $A C_{*}$ on subset $E$ of $[a, b]$ if the sum of its oscillations $\Sigma \omega\left(F ; I_{k}\right)$, over non-overlapping subintervals with endpoints in $E$, converges to zero uniformly as the sum of the lengths of the $I_{k}$ goes to zero; a sequence $\left\{F_{n}\right\}$ is equi- $A C_{*}$ if the convergence is also uniform in $n$ (both properties pass to subsets); $F$ is $A C G_{*}$ and $\left\{F_{n}\right\}$ equi- $A C G_{*}$ if $[a, b]$ is the union of a sequence of such subsets. These entail the ordinary (unstarred) $A C$ properties, in which $\Sigma \omega\left(F ; I_{k}\right)$ is replaced by $\Sigma\left|F\left(I_{k}\right)\right|$, the sum of absolute values of differences of $F$ at the endpoints of $I_{k}$; and entail, further, absolute continuity of the (outer)

Mathematical Reviews subject classification: Primary: 26A39. Secondary: 26A46

Received by the editors December 22, 1995 
measure induced on $E$ by the increment of $F$ over intervals with endpoints in $E$ - resp. equi-absolute continuity for an equi- $A C$ sequence.

The classical Vitali convergence theorem on an interval reads: If Lebesgue integrable (i.e. "summable") $f_{n}$ converge in measure to $f$ and their primitives $F_{n}$ are equi- $A C$ then $f$ is integrable to the limit of the $F_{n}$. (See [N, VI.§3, Theorem 2] for the theorem and [S, III.§12] for the coincidence of the two notions of absolute continuity). The hypothesis is in some sense stronger than that above in requiring an absolute continuity on the whole interval rather than in the generalized sense; but so is the conclusion in that the convergence of the $F_{n}$ is deduced rather than postulated.

The best known recent Henstock convergence theorem is the "controlled" convergence theorem of Lee and Chew [LC]: This requires a.e. convergence of $f_{n}$ to $f$ and, in addition to equi- $A C G_{*}$ of the $F_{n}$, their uniform, rather than pointwise, convergence. The latter was subsequently improved to pointwise convergence to a continuous function as above, by Liao Ke-Cheng [L], but still retains the stronger a.e. convergence of the integrands and the proof, starting from the original controlled convergence theorem as a given, implicitly requires the somewhat elaborate development in [LC].

The proof which follows owes a good deal to the analysis carried out by Gordon in the recent $[\mathrm{G}]$.

It will suffice to show that on each of a countable sequence of subsets $B$ which cover $[a, b]$ there is a positive function $\delta$ such that $\mid \Sigma f\left(t_{i}\right)\left(v_{i}-u_{i}\right)-$ $F\left(v_{i}\right)+F\left(u_{i}\right) \mid \equiv *$ is small for all $\delta$-fine partial divisions tagged in $B$ : for then by disjointifying the $B$ and gluing together $\delta$ 's which make $*<\varepsilon / 2^{n}$ on the $n^{\text {th }} B$ one obtains a $\delta$ on $[a, b]$ for whose $\delta$-fine divisions $\mid \Sigma f\left(t_{i}\right)\left(v_{i}-u_{i}\right)-$ $F(b)+F(a) \mid<\varepsilon$.

On subsets $C$ on which the $F_{n}$ are equi- $A C_{*}$ (which may by their continuity be taken closed) the $F_{n}$ are restrictions of equi- $A C$ Lebesgue integrals (e.g. by extending the $F_{n}$ to the open complement of $C$ by making them linear in each contiguous subinterval and continuous at its endpoints) hence, by the classical Vitali Theorem (which furnishes $L_{1}$ convergence) $\sum F_{n}\left(v_{i}\right)-F_{n}\left(u_{i}\right)$ converges in $n$ uniformly on the partial divisions with endpoints in $C$.

Write $C$ as a countable union of $B$ 's on each of which $f$ is bounded. By convergence in measure there is in each $B$ a relatively open subset $U$, thus a countable union of portions, on whose complement $f$ is uniformly close to an $f_{N}$ with index so large that $\sum F_{N}\left(v_{i}\right)-F_{N}\left(u_{i}\right)$ is uniformly close to $\sum F\left(v_{i}\right)-F\left(u_{i}\right)$, and whose measure times the bound of $f$ on $B$ is small, as well as the sum of the oscillations of each of the $F_{n}$ (hence also of $F$ ) over non-overlapping subintervals with endpoints in $U$, by equi- $A C_{*}$. Define $\delta$ : for the internal points of each portion, to be less than the distance to enclosing 
points in it; at every extreme point, so that differences of the continuous $F$ in its $\delta$-neighborhood and the bound of $f$ times $\delta$ are so small that summed over the at most countably many such points they are small, and elsewhere so as to also make $\sum f_{N}\left(t_{i}\right)\left(v_{i}-u_{i}\right)$ close to $\sum F_{N}\left(v_{i}\right)-F_{N}\left(u_{i}\right)$ for all $\delta$-fine partial divisions tagged in $B$, by Henstock's Lemma.

Then $*$ is small for $\delta$-fine partial divisions tagged in $B$ because each of the two summed terms is individually small for the partial subdivision tagged in $U$ while the summed difference is small for the part tagged in the complement of $U$.

\section{References}

[G] Russell A. Gordon, A general convergence theorem for non-absolute integrals, J. London Math. Soc., 2, no. 44 (1991), 301-309.

[L] Ke Cheng Liao, A Refinement of the controlled convergence theorem for Henstock integrals, Southeast Asian Bulletin of Mathematics, 11(1) (1987), 49-51.

[L'] Peng-Yee Lee, Lanzhou Lectures on Henstock Integration, World Scientific, Singapore, 1989.

[LC] Peng-Yee Lee and T. S. Chew, A better convergence theorem for Henstock integrals, Bull. London Math. Soc., 17 (1985), 557-564.

[LC'] Peng-Yee Lee and T. S. Chew, A short proof of the controlled convergence theorem for Henstock integrals, Bull. London Math. Soc., 19 (1987), 60-62.

[N] I. P. Natanson, Theory of functions of a real variable, Vol. 1, Ungar, New York, 1964.

[S] S. Saks, Theory of the integral, 2nd edition revised, Hafner Publications, New York, 1937. 